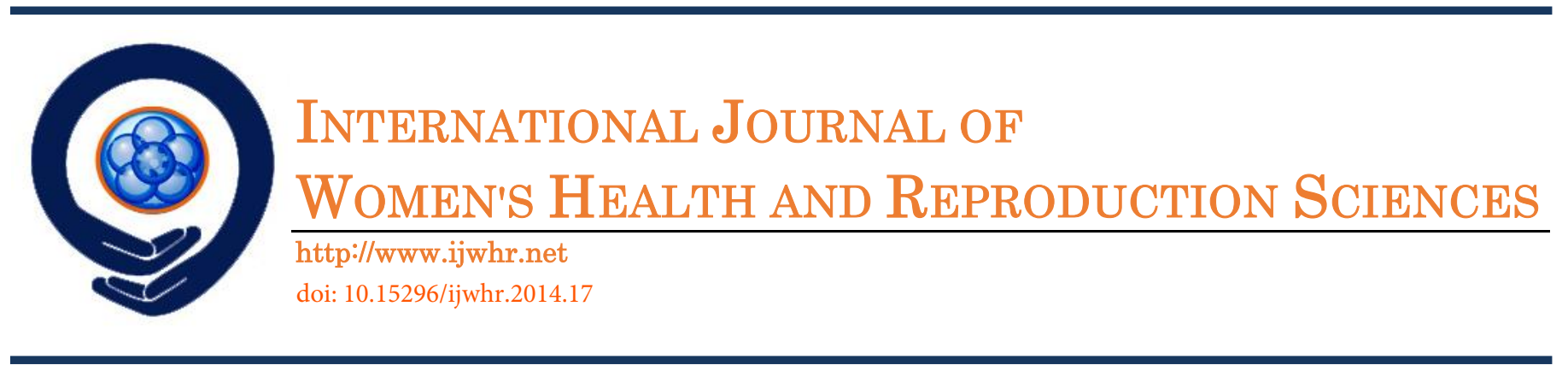

\title{
Pathogenic Effects of Genital Schistosomiasis on Men and Women Health
}

\author{
Yagoob Garedaghi ${ }^{*}$
}

Article History:
Received 22 October 2013
Accepted 3 February 2014
Revised 22 January 2014
Available online 4 February 2014
Keywords:
Genital Schistosomiasis
Men and women
Pathogenic Effects
Corresponding Author:
Yagoob Gharedaghi, Assistant professor, PhD,
Department of Veterinary Parasitology,
Tabriz Branch, Islamic Azad University,
Tabriz, Iran.
Tel: +989143110499
Email: y_garedaghi@yahoo.com

\section{Dear Editor:}

Genital Schistosomiasis (GS) is a disease condition of the genitalia caused by invasion of reproductive tissues by Schistosoma ova. This health condition has been associated with reproductive health problems such as ectopic pregnancies, postcoital bleeding, bleeding, low sperm count, cervical cancer and found to increase the risk of acquiring sexually transmitted disease(STD) including HIV/AIDS (1). According to the worldwide estimate of women with this condition, Female Genital Schistosomiasis (FGS) is in the range of 2-13 million (2). Studies have shown that FGS is a common manifestation in S. haematobium infections; hence, a strong association has been observed between urinary schistosomiasis and genital schistosomiasis (3). Genital schistosomiasis (GS), a clinical manifestation of schistosomiasis is likely to occur in all adult cases of schistosomiasis with debilitating disease sequelae. Studies have shown a high prevalence of genital schistosomiasis in communities with endemic schistosomiasis(3). Schistosomiais is prevalent in tropical and sub-tropical areas, especially in poor communities without access to safe drinking water and adequate sanitation. It is estimated that at least $90 \%$ of those requiring treatment for schistosomiasis live in Africa (4). There are two major forms of schistosomiasis intestinal and urogenital - caused by five main species of blood fluke (Table 1).

Schistosomiasis particularly affects populations involving in agricultural and fishing activities. Women doing domestic chores in infested water such as washing clothes, are also at risk. Inadequate hygiene and play habits make children especially vulnerable to infection (Figure.1).

Migration to urban areas and refugee movements are introducing the disease to new areas. Increasing population size and the corresponding needs for power and water often result in development schemes and environmental modifications that also lead to increased transmission (5).With the rise in eco-tourism and travel "off the beaten track", increasing numbers of tourists are contracting schistosomiasis. At times, tourists present with severe acute infection

1- Assistant professor, PhD, Department of Veterinary Parasitology, Tabriz Branch, Islamic Azad University, Tabriz, Iran. 
and unusual problems, including paralysis $(6,7)$.

Urogenital schistosomiasis is also considered to be a risk factor for HIV infection, especially in women (8). The tissue around both viable and dead S.haematobium eggs has increased vascularity and a high density of macrophages, lymphocytes, foreign body giant cells, eosinophils, neutrophils, plasma cells, Langerhans cells, fibroblasts, and multinucleated histiocytes $(8,9)$. Even calcified eggs may induce the influx of immune cells and blood vessel proliferation, local bleeding and edema (10).

Symptoms of schistosomiasis are caused by the body reaction to the worm eggs, not by the worms themselves. Intestinal schistosomiasis can result in abdominal pain, diarrhea, and blood in the stool. Liver enlargement is common in advanced cases, and is frequently associated with an accumulation of fluid in the peritoneal cavity and hypertension of the abdominal blood vessels. In such cases, there may also be enlargement of the spleen (9).

The classic sign of urogenital schistosomiasis is haematuria (blood in urine). Fibrosis of the bladder and ureter, and kidney damage are sometimes diagnosed in advanced cases. Bladder cancer is another possible late-stage complication. In women, urogenital schistosomiasis may have some symptoms, including genital lesions, vaginal bleeding, dyspareunia (pain during sexual intercourse) and nodules in the vulva. In men, urogenital schistosomiasis can induce pathology of the seminal vesicles, prostate and other organs. This disease may also have other long-term irreversible consequences, including infertility.

The economic and health effects of schistosomiasis are considerable. In children, schistosomiasis can cause anemia, stunting and a reduced ability to learn, although the effects are usually reversible with treatment. Chronic schistosomiasis may affect personal ability to work and in some cases can result in death. In subSaharan Africa, more than 200000 deaths per year are due to schistosomiasis (10).

Schistosomiasis is diagnosed through the detection of parasite eggs in stool or urine specimens. Antigens detected in blood or urine samples are also indications of infection.

For urogenital schistosomiasis, a filtration technique using nylon, paper or polycarbonate filters is the standard diagnostic technique. Children with $\mathrm{S}$. haematobium almost always have microscopic blood in their urine and this can be detected by chemical reagent strips.

The eggs of intestinal schistosomiasis can be detected in faecal specimens through a technique using methylene blue-stained cellophane soaked in glycerine or glass slides, known as the Kato-Katz technique.

For people living in low transmission or nonedemic areas, serological and immunological tests may be useful in showing exposure to infection and the need for thorough examination and treatment (11).

The control of schistosomiasis is based on large-scale treatment of at-risk population groups, access to safe water, improved sanitation, hygiene education and snail control.

The WHO strategy for schistosomiasis control focuses on reducing disease through periodic and targeted treatment with Praziquantel. This involves regular treatment of all people in at-risk groups.

Groups targeted for treatment are:

- School-aged children in endemic areas

- Adults considered to be at risk in endemic areas, people with occupations involving in contact with infested water - such as fishermen, farmers, irrigation workers and women whose domestic tasks bring them into contact with infested water

- Entire communities living in highly endemic areas

The frequency of treatment is determined by the prevalence of infection or haematuria (in the case of urogenital schistosomiasis) in school-age children. In high transmission areas, treatment may have to be repeated every year for a number of years. Monitoring is essential to determine the efficiency of control interventions (12).

The aim of these interventions is to reduce disease; periodic treatment of at-risk populations improves mild symptoms and prevents infected people from developing severe and late-stage chronic disease. 
However, a major limitation to schistosomiasis control has been access to Praziquantel. Available data show that only $10 \%$ of people requiring treatment were reached in 2011.

Praziquantel is the recommended treatment against all forms of schistosomiasis. It is effective, safe and low-cost. Even though reinfection may occur after treatment, the risk of developing severe disease is diminished and even reversed when treatment is initiated and repeated in childhood.

Schistosomiasis control has been successfully implemented over the past 20 years in several countries, including Brazil, Cambodia, China, Egypt, and Saudi Arabia. There is evidence that schistosomiasis transmission was interrupted in Morocco. Assessment of the status of transmission is being made in several countries.

Over the past 10 years, there has been scale up of treatment campaigns in a number of sub-Saharan countries, with national coverage achieved in Burkina Faso, Niger and Uganda.

In 2011, reports were received from 24 countries on preventive chemotherapy for schistosomiasis. The reported number of people treated for schistosomiasis increased from 12.4 million in 2006 to 28.1 million in 2011, down from 33.5 million in 2010 representing a decrease of $20 \%$ in the number of treated people. This was due to logistic reasons, unreliable funding for implementation, change of implementing contractors, inadequate capacity at country level, fewer countries reporting data and also fewer people treated in some countries which reported data (13).

WHO work on schistosomiasis is part of an integrated approach to the control of neglected tropical diseases. Although medically divers, neglected tropical diseases share features that allow them to persist in conditions of poverty, where they cluster and frequently overlap. WHO coordinates the strategy of preventive chemotherapy in consultation with collaborating centres and partners from academic and research institutions, the private sector, nongovernmental organizations, international development agencies and other United Nations organizations. WHO develops technical guidelines and tools for use by national control programs.

Working with partners and the private sector, WHO has advocated for increased access to Praziquantel and resources for implementation. A significant amount of Praziquantel, to treat more than 100 million children of school age per year has been pledged by the private sector and development partners (14).

\section{Acknowledgement:}

The author would like to thanks Tabriz Branch, Islamic Azad University for the informational and scientific supports of this research.

Table 1. Parasite species and geographical distribution of schistosomiasis

\begin{tabular}{|c|c|c|}
\hline Forms of schistosomiasis & Species & Geographical distribution \\
\hline \multirow{4}{*}{ Intestinal schistosomiasis } & Schistosoma mansoni & $\begin{array}{l}\text { Africa, the Middle East, the } \\
\text { Caribbean, Brazil, } \\
\text { Venezuela, Suriname }\end{array}$ \\
\hline & Schistosoma japonicum & $\begin{array}{l}\text { China, Indonesia, the } \\
\text { Philippines }\end{array}$ \\
\hline & Schistosoma mekongi & $\begin{array}{l}\text { Several districts of } \\
\text { Cambodia and the Lao } \\
\text { People's Democratic } \\
\text { Republic }\end{array}$ \\
\hline & $\begin{array}{l}\text { Schistosoma guineensis and } \\
\text { related S. intercalatum }\end{array}$ & $\begin{array}{l}\text { Rain forest areas of central } \\
\text { Africa }\end{array}$ \\
\hline Urogenital schistosomiasis & Schistosoma haematobium & Africa, the Middle East \\
\hline
\end{tabular}




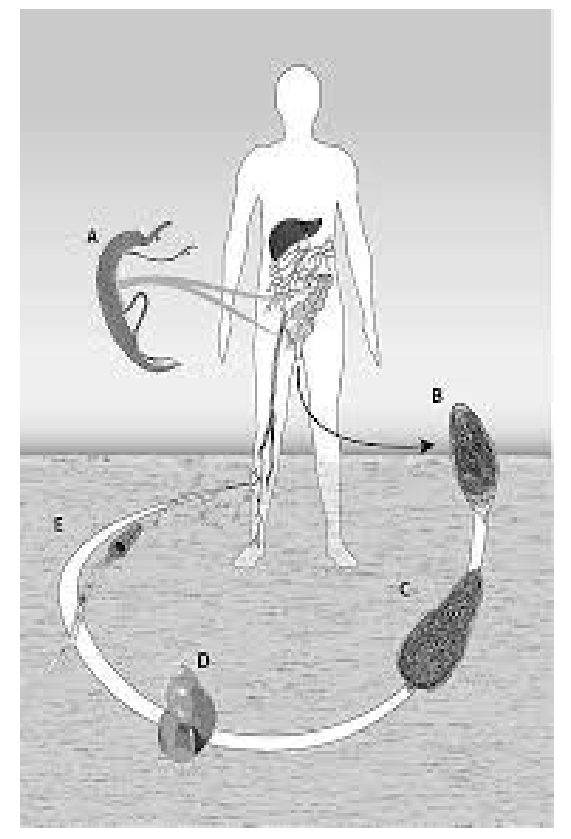

Figure 1. Life cycle of Schistosoma Haematobium

\section{References:}

1. World Health Organization. Schistosomiasis, fact sheet No. 115, 2012. Available at: http://www.who.int/mediacentre/factsheets/fs115/en/ index.html. Accessed 26 August 2012.

2. Poggensee G, Feldmeier H. Female genital schistosomiasis: facts and hypotheses. Acta Trop. 2001; 79:193-210.

3. Kjetland EF, Leutscher PD, Ndhlovu PD. A review of female genital schistosomiasis. Trends Parasitol. 2012; 28:58-65.

4. Adegnika AA, Ramharter M, Agnandji ST, Ateba Ngoa U, Issifou S, Yazdanbaksh M, et al. Epidemiology of parasitic co-infections during pregnancy in Lambaréné, Gabon. Trop Med Int Health. 2010; 15:1204-9.

5. Ramharter M, Adegnika AA, Agnandji ST, Matsiegui PB, Grobusch MP, Winkler S, et al. History and perspectives of medical research at the Albert Schweitzer Hospital in Lambaréné, Gabon. Wien Klin Wochenschr. 2007; 119:8-12. 
6. Chandiwana SK, Woolhouse ME, Bradley M. Factors affecting the intensity of reinfection with Schistosoma haematobium following treatment with praziquantel. Parasitology. 1991; 102:73-83.

7. Hagan P. Reinfection, exposure and immunity in human schistosomiasis. Parasitol Today. 1992; 8:12-6.

8. Swai B, Poggensee G, Mtweve S, Krantz I. Female genital schistosomiasis as an evidence of a neglected cause for reproductive ill-health: a retrospective histopathological study from Tanzania. BMC Infect Dis. 2006; 6:134.

9. Mbabazi PS, AndanO, Fitzgerald DW, ChitsuloL, EngelsD, Downs JA. Examining the relationship between urogenital schistosomiasis and HIV infection. PLoS Neglected Tropical Diseases. 2011;(12):e1396.

10. Kjetland EF, Ndhlovu PD, Gomo E, Mduluza T, Midzi N, Gwanzura L, et al. Association between genital schistosomiasis and HIV in rural Zimbabwean women. AIDS. 2006 ; 28; 20(4):593-600.

11. Poggensee G,Feldmeier H. Female genital schistosomiasis:facts and hypothesis. Actatropica. 2001; 79:193-210.

12. Leutscher P, Ravaolimalala C, Raharisolo CE, Ramarokoto M, Rasendramino A, Raobelison B, Vennervald PE,Feldmeir H. Clinical findings in female genital schistosomiasis in Madagascar. Tropical Medicine and International Health. 1998;3(4):327-33.

13. Leutscher P, Ramarokoto CE, Reimert C, Feldmeier H, Esterre P, Vennervald BJ. Community-based study of genital schistosomiasis in men from Madagascar. Lancet. 2000; 8(9198):117-118.

14. Richens J. Genital manifestations of tropical diseases. Sexually Transmitted Infections. 2004;80(1):12-17. 